\title{
Orthonormal Method for Compact Global Navigation Satellite Systems Antenna Array Designs
}

\author{
Ezequiel A. Marranghelli \\ Ramón López La Valle \\ Pedro A. Roncagliolo \\ SENyT, Departamento de Electrotecnia, Facultad de Ingenierí, Universidad Nacional de La Plata, Argentina.
}

\begin{abstract}
Compact antenna arrays require special considerations at design time not to degrade its efficiency and also the spatial diversity becomes reduced in terms of phase resolution as distance diminishes. Additionally, element radiation patterns are affected and the objective of equally behaved antennas is even more difficult to achieve. However, the induced antenna gain differences can actually be helpful because they can be used to maintain the degrees of freedom in array radiation pat-tern subspace. In this article, a general analysis on the relationship of efficiency and beam patterns are reviewed to prove that maximum efficiency is achievedwhen radiation patterns fulfill a given orthogonality condition. Under this condition, we propose to apply a unitary matrix transformation to the array response thatcan translate the compact antenna array behavior into the equivalent response of a ${ }_{2}^{\lambda}$-spaced ideal antenna array without modifying its total efficiency. Consequently, an useful compact antenna array should be designed to have orthogonal radiation beams. Then, applying the transformation, they can be translated to another orthogonal radiation patterns set with only phase differences and a common gain pattern. Simulated results of a computer-aided design of a practical array of Global Navigation Satellite Systems microstrip antennas are presented.
\end{abstract}

\section{Keywords}

Compact microstrip array, efficiency, orthonormal transformation, reconfigurablepatterns, smart antennas

\section{INTRODUCTION}

Global Navigation Satellite Systems (GNSS) have become the standard choice for obtaining position, velocity, and time of any vehicle or structure equipped with a receiver. ${ }^{1}$ Arrays of identical antennas are currently proposed in many GNSS applications for increasing visibility and improving received signal quality maintaining, at the same time, robustness in diverse and challenging scenarios where undesired perturbations as interferences, jamming, spoofing signals, or even multipath could be potential threats that severely degrade the GNSS performance. An antenna array (AA) provides spatial diversity, that is, the ability of discriminating incoming signals based on the characteristics of the generated radiation pattern (RP) subspace. ${ }^{2}$ Signal discrimination based on its direction of arrival (DOA) is one of the typical applications allowed by the use of AAs.

Nowadays miniaturization is also an essential requirement, therefore small arrays using a reduced number of antennas are highly desired. ${ }^{3-5}$ However, in such arrays the influence of mutual coupling and electromagnetic diffraction (MCED) is unavoidable. ${ }^{6}$ These phenomena have several effects in terms of efficiency and final response of individual RPs, both strongly related to each other. ${ }^{7,8}$ The so-called in situ RPs are the resultant individual RPs when embedded in the array and their surrounding electromagnetic environment. From classical theory of phased arrays with equally behaved antennas, spatial diversity is provided by their 
relative positions so that signals arriving from all possible DOAs can be discriminated by phase differences according to each traveling path length. ${ }^{9}$ In that case, the optimal distance between elements is $\frac{\lambda}{2}$, being $\lambda$ the carrier wavelength of the traveling wave, because it provides maximum phase resolution and no ambiguity. However, this ideal situation is altered when considering actual in situ RPs, and even more when arrays with less than $\frac{\lambda}{2}$ inter-element distances are employed in compact designs. If we recall the case of two equally behaved antennas in terms of their RPs, separated by a distance of $\frac{\lambda}{2}$, the maximum range of phase differences they can measure at carrier central frequency is $\left[-\frac{\pi}{2}, \frac{\pi}{2}\right]$. The same AA but separated a distance $d<\frac{\lambda}{2}$ can only measure phase differences in the range $\left[-\frac{\pi d}{\lambda}, \frac{\pi d}{\lambda}\right]$ which means narrower resolution restricted by their relative positions. But this is not always the case, as a particular coupled AA design could actually improve performance. ${ }^{10}$ This result suggest that proper designs for RPs can represent a benefit in terms of efficiency and DOA discrimination, even though it also implies more signal processing complexity because the common RP assumption is no longer valid. Indeed, it is an advantage to exploit gain and phase differences in a joint manner at the array design. Otherwise, the spatial diversity could be degraded and the efficiency greatly reduced in compact arrays.

Many compensation methods have been proposed to reduce differences on the in situ RPs. ${ }^{11-14}$ However, they do not account for the effects of antenna thermal noise. For a multiantenna GNSS receiver, each channel/antenna signal is essentially immersed into thermal noise that typically can be considered independent and identically distributed (i.i.d.) among them. After applying a given pattern compensation algorithm, the signal coming from each antenna element seems to be received by an ideal phased array, but the noise distribution becomes distorted. However, i.i.d. noise assumption is needed or desired in most of the classical theory of phased arrays signal processing. Hence, to retain the advantage of i.i.d noise after a transformation, the compensation method must satisfy the restriction of being not only linear but also orthonormal. In this work a unitary matrix transformation method for AAs is presented which is based on wellknown solution of the orthogonal Procrustes problem (OPP) posed in Reference 15 . The fundamental virtue of a unitary transformation is that delivers a new "virtual" array without altering any signal or noise power correlation structure. Then, our method provides an antenna behavior compensation to translate the in situ RPs into an equivalent AA of equally behaved elements. Therefore, a particular array design that has the ability of perceiving gain and phase differences can be translated into an equivalent AA with almost identical RPs. In particular, we present a simulated example of microstrip antennas forming a compact array that can behave similar to a $\frac{\lambda}{2}$-spaced AA whose elements have a common RP. It is worth mentioning that the designed array performs efficiently without needing any additional absorber material, resonant structure, fences, or any decoupling and matching network stages. ${ }^{4,5,16-18}$ The proposed method intends to turn many efficient coupled AA designs workable with classic phased array techniques.

The rest of the article is organized as follows. In Section 2, received signals structure along with its parameters of interest are described. AA efficiency is also considered and related to in situ RPs. In Section 3, the unitary transformation method is defined and characterized for optimal array compensation. In Section 4, a practical GNSS microstrip array design is presented and evaluated after applying the orthonormal compensation method. Finally, in Section 5, relevant conclusions are stated.

\section{RECEIVED SIGNAL MODEL}

Consider an array of $N$ identical antennas for GNSS signals. Thus, the $n$th in situ antenna RP centered at $\left(x_{n}, y_{n}, z_{n}\right)$ position and for a given $(\theta, \varphi)$ direction can be calculated as. ${ }^{9}$

$$
h_{n}(\theta, \varphi)=s_{n}(\theta, \varphi) g_{n}(\theta, \varphi),
$$

where $\theta$ and $\varphi$ represent elevation and azimuth coordinates; $g_{n}$ is the $n$th embedded far-field gain pattern centered at its own geometric center:

$$
\because \mathrm{g}_{\mathrm{n}}(\theta, \varphi)=\left|\mathrm{g}_{\mathrm{n}}(\theta, \varphi)\right| \angle \mathrm{g}_{\mathrm{n}}(\theta, \varphi),
$$

$s_{n}$ is the $n$-th element of the steering vector that is unitary and phase dependent on the geometry of the array:

$$
\because \mathrm{s}_{\mathrm{n}}(\theta, \varphi)=\mathrm{e}^{\mathrm{j} \frac{2 \pi}{\lambda}\left(\mathrm{x}_{\mathrm{n}} \sin (\theta) \cos (\varphi)+\mathrm{y}_{\mathrm{n}} \sin (\theta) \sin (\varphi)+\mathrm{z}_{\mathrm{n}} \cos (\theta)\right)}
$$

where $\left(x_{n}, y_{n}, z_{n}\right)$ are the coordinates of the geometric centers of each antenna referred to the geometric center of the array, that is, $(0,0,0)$.

After reception by the antenna elements, the signal is preamplified, band-limiting filtered, down-converted to baseband, and digitized. If a narrowband signal model is considered, the AA, front-end and analog to digital converters stages can be modeled as a flat frequency response system. ${ }^{19}$ Employing complex envelope notation for $P$ incident signal sources, the received baseband digital signal over each antenna at $m$ th snapshot can be expressed as. ${ }^{20}$

$$
x_{n}[m]=\sum_{p=1}^{P} \mathrm{y}_{p}[m] h_{n}\left(\theta_{p}, \phi_{p}\right)+\mathrm{e}_{n}[m],
$$

where $x_{n}$ is the measured signal amplitude by the $n$th antenna; $y_{p}$ is the digitized incoming signal amplitude by the $n$th antenna from far-field orientation $\left(\theta_{p}, \varphi_{p}\right)$, for $p=1, \ldots, P ; \mathrm{e}_{n}$ is the total received thermal noise by $n$-th antenna and their corresponding front-end.

In matrix notation, the measurement vector can be expressed as 


$$
\mathbf{x}[m]=(S \circ G) \mathbf{y}[m]+\mathbf{e}[m]=H \mathbf{y}[m]+\mathbf{e}[m],
$$

with $[\mathbf{x}]_{N \times 1},[\mathbf{y}]_{P \times 1},[\mathbf{e}]_{N \times 1}$, $\circ$ denotes the Hadamard product and

$$
\begin{aligned}
S=\left[\mathbf{s}_{1}, \ldots, \mathbf{s}_{\mathrm{P}}\right] & =\left[\begin{array}{ccc}
s_{1,1} & \cdots & s_{1, P} \\
\vdots & \cdots & \vdots \\
s_{N, 1} & \cdots & s_{N, P}
\end{array}\right] ; s_{n, p}=s_{n}\left(\theta_{p}, \phi_{p}\right), \\
G=\left[\mathbf{g}_{1}, \ldots, \mathbf{g}_{\mathrm{P}}\right] & =\left[\begin{array}{ccc}
g_{1,1} & \cdots & g_{1, P} \\
\vdots & \cdots & \vdots \\
g_{N, 1} & \cdots & g_{N, P}
\end{array}\right] ; g_{n, p}=g_{n}\left(\theta_{p}, \phi_{p}\right), \\
H=\left[\mathbf{h}_{1}, \ldots, \mathbf{h}_{\mathrm{P}}\right] & =\left[\begin{array}{ccc}
h_{1,1} & \cdots & h_{1, P} \\
\vdots & \cdots & \vdots \\
h_{N, 1} & \cdots & h_{N, P}
\end{array}\right] ; h_{n, p}=h_{n}\left(\theta_{p}, \phi_{p}\right),
\end{aligned}
$$

where $H$ is called the array matrix response.

In the following, we focus on the definition of AA efficiency and how it reveals desired characteristics of compact arrays. This is strongly related to its RPs and the obtained spatial diversity.

\subsection{AA efficiency}

For the sake of simplicity, the next analysis will be done in transmission mode. In fact, for a reciprocal antenna system, the Maxwell-Lorentz Electromagnetic Reciprocity Theorem establishes the equivalence principle between radiating patterns in transmitting and receiving mode as well as all characteristic parameters of a multi-port system. ${ }^{7}$

The total delivered power to the radiating array by applied signal vector $\mathrm{x}$ is

$$
P_{\text {total }}=x^{H} x .
$$

The AA system, constitutes the physical medium where radiation takes place. However, not all delivered power translates into radiated power, because some of it can be reflected at the channel ports and some is dissipated in dielectric and metallic materials as ohmic losses, then, by the Principle of Conservation of the Energy, it can be stated that. ${ }^{7}$

$$
P_{\text {total }}-P_{\text {refl }} \geq P_{\text {rad }},
$$

that provides a practical limit to the total radiated power. It can be further expressed as

$$
x^{H}\left(I_{N}-S^{H} S\right) x \geq x^{H} \Gamma x,
$$

where $S$ is the scattering parameters matrix and $\Gamma$ is the radiation power efficiency and distribution matrix. In previous equations, equality holds if and only if all of the radiating system can be assumed as a lossless structure.

Radiation matrix elements $\Gamma_{i k}$ can be expressed in terms of antenna efficiencies and the beam coupling factors as. ${ }^{7,9}$

$$
\Gamma_{i, k}=\epsilon_{i}^{*} \eta_{i}^{*} \beta_{i, k} \epsilon_{k} \eta_{k},
$$

where $\epsilon_{i}\left(\epsilon_{k}\right)$ stands for the $i$ th $(k$ th) antenna reflection efficiency, $\eta_{i}\left(\eta_{k}\right)$ stands for the $i$ th $(k$ th) antenna radiation efficiency, superscript $*$ denotes complex conjugate, and

$$
\beta_{i, k}=\oint \check{h}_{i}(\theta, \varphi) \check{h}_{k}^{*}(\theta, \varphi) \sin (\theta) d \theta d \varphi,
$$

defines the beam coupling factor between $i$ th and $k$ th antenna responses. The RP $\breve{h}_{i}(\theta, \varphi)$ is the $\check{h}_{i}(\theta, \varphi)$ defined in Equation 1 but normalized so that $\beta_{i, i}=1$.

The amount $1-\left|\eta_{i}\right|^{2}$ is the proportion of power that is lost by ohmic losses in metallic and dielectric materials given by their geometry and finite conductivity. Similarly, $1-\left|\epsilon_{i}\right|^{2}$ is the relative amount of power that is reflected out of the antenna system at channel ports, that is,

$$
\left|\epsilon_{i}\right|^{2}=1-\sum_{k=1}^{N}\left|S_{i, k}\right|^{2} .
$$

Therefore, from Equations 6 to 9, the following conclusions about particular cases can be enounced:

- If $S^{H} S$ is not a diagonal matrix, then the reflection efficiency is limited by reflection and coupling losses.

- If $S^{H} S$ is a diagonal matrix with nonzero elements, then it is verified that $\beta_{i . k}$ form an identity matrix and $\epsilon_{i}<1$, for some $i$ index in $i=1, \ldots, N$.

- If $S^{H} S$ is a completely null matrix, no reflection losses exist.

In consequence, a completely matched and lossless system needs $\epsilon_{i}=\eta_{i}=1$, with $i=1, \ldots, N$, besides $\beta_{i, k}=0$, with $i \neq k$. Then, it is straightforward to conclude that an ideal radiation structure must provide $N$ orthogonal beam patterns by means of $N$ perfectly matched ports without mutual coupling losses. This condition seems more easily achievable for large arrays, but not a simple objective for compact arrays where MCED is unavoidable.

The following examples present simple arrays, whose RPs are actually orthogonal, and that meet the desired characteristics for efficient radiation.

\subsection{Example 1}

First we consider the classic and ideal linear array of $N$ isotropic antennas, being $N$ an even number, whose gain amplitude is normalized to 1 for single frequency operation at wavelength $\lambda$. Antennas are aligned along the $x$-axis, centered at the origin of coordinates, and separated by a distance $d$ between them. In Figure 1, an illustrative scheme is presented where the dependency of the variable $\varphi$ is omitted for the sake of simplicity.

It is a phased array with identical elements where an array factor of constant amplitude is the common term as the 


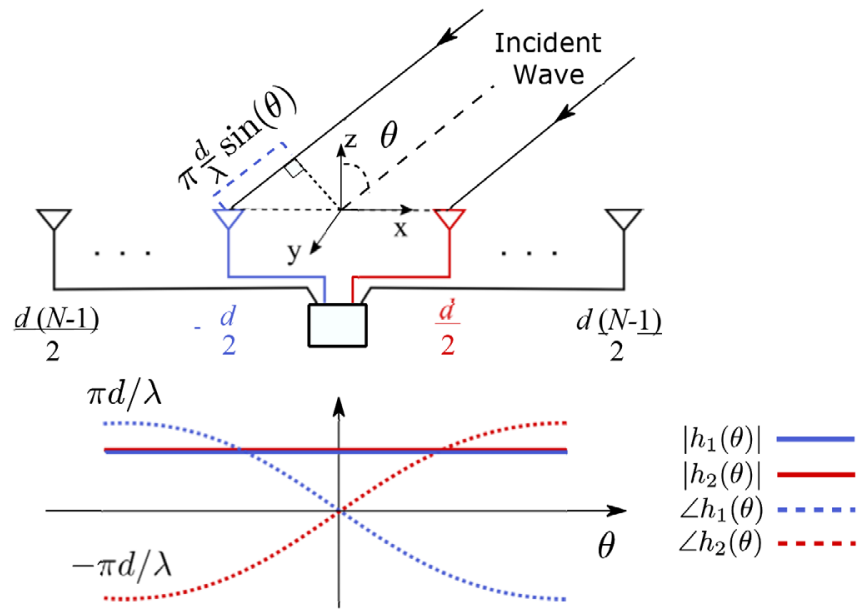

FIGURE 1. Phased antenna array

RP vector. The only change is the phase dependency according to the signal orientation. Then, the RP vector results

$$
\mathbf{h}(\theta, \phi)=\left[\begin{array}{c}
e^{j \frac{2 \pi}{\lambda}\left(\left(-(N-1) \frac{d}{2}\right) \sin (\theta) \cos (\phi)\right)} \\
e^{j \frac{2 \pi}{\lambda}\left(\left(-(N-3) \frac{d}{2}\right) \sin (\theta) \cos (\phi)\right)} \\
\vdots \\
e^{j \frac{2 \pi}{\lambda}\left(\left(+(N-1) \frac{d}{2}\right) \sin (\theta) \cos (\phi)\right)}
\end{array}\right]
$$

If no reflection losses can be assumed, it can be verified from Equation 8 that the desired characteristics for $\Gamma$ matrix are met when $d=\frac{\lambda}{2}$, yielding

$$
\beta_{i, k}=\oint \frac{e^{j \pi \sin (\theta) \cos (\phi)(i-k)}}{4 \pi} \sin (\theta) d \theta d \varphi=\left\{\begin{array}{c}
1 i=k \\
0 i \neq k
\end{array}\right.
$$

It should be noted that not only phased arrays can achieve this condition. We consider next another example where the antennas share the same phase center and, for instance, signals always arrive without relative phase differences.

\subsection{Example 2}

The crossed-loop antennas, like the crossed-dipole antennas, is another simple case of an AA. ${ }^{21}$ It was very helpful at the beginning of the XX century as an analog system for direction finding, and its built-in structure to this application was known as the Watson-Watt Direction Finder. A simplified scheme is shown in Figure 2. It contains two crossed-loop antenna with approximated RP given by $\cos (\varphi)$ and $\sin (\varphi)$ functions when described over $x y$-plane. At this system, each antenna is connected to a receiver and both channel signals are simultaneously displayed on a cathode ray tube as a $X Y$ graph so that an impinging wave on the array can be estimated with the bearing angle of resulting graph. Given the azimuth ambiguity of $\pi$ radians, bearing angle can only be discriminated without ambiguity at a reduced range, that is, $-\frac{\pi}{2} \leq \phi \leq \frac{\pi}{2}$. It can also be realized that the magnitude of the RPs changes according orientation, but not its phase. Therefore, it can be said that their phase center is shared and it is located at the geometric center of the loop array.

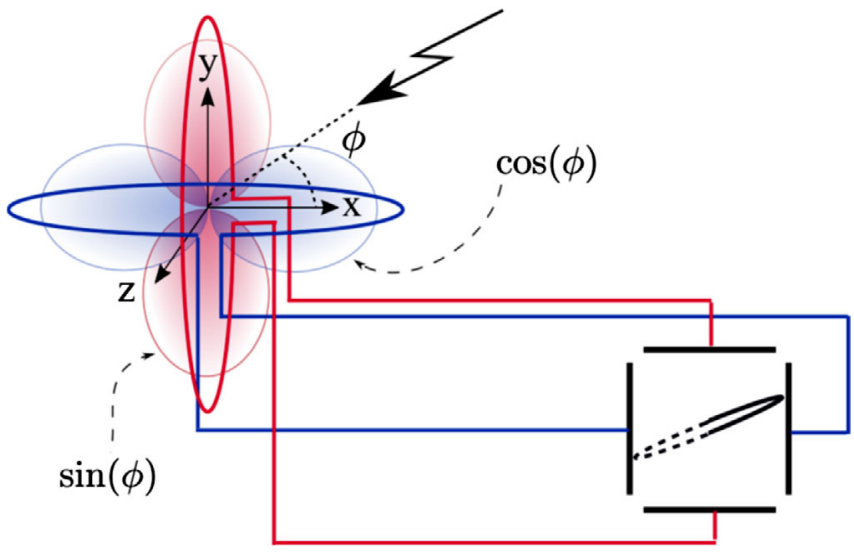

FIGURE 2. Crossed-loop antennas direction finding system

As an AA, its performance will be analyzed in terms of its efficiency and compared to an equivalent design based on equally behaved antennas.

The RP vector of the crossed-loop antennas to a given $\operatorname{DOA}(\theta, \varphi)$ is

$$
\mathbf{h}(\theta, \phi)=\left[\begin{array}{c}
\cos \left[\tan ^{-1}\left(\frac{\left(\cos ^{2} \theta+\sin ^{2} \theta \sin ^{2} \phi\right)^{\frac{1}{2}}}{\sin \theta \cos \phi}\right)\right] \\
\sin \left[\tan ^{-1}\left(\frac{\left(\cos ^{2} \theta+\sin ^{2} \theta \sin ^{2} \phi\right)^{\frac{1}{2}}}{\sin \theta \cos \phi}\right)\right]
\end{array}\right] .
$$

Hence, from Equation 8 the $\beta_{i k}$ elements

$$
\beta_{i, k}=\oint \check{h}_{i}(\theta, \varphi) \check{h}_{k}^{*}(\theta, \varphi) \sin (\theta) d \theta d \varphi=\left\{\begin{array}{c}
1 i=k \\
0 i \neq k
\end{array}\right.
$$

which is the same beam coupling matrix that could be achieved with a $\frac{\lambda}{2}$ separated pair of ideal isotropic antennas, as shown in the previous example, taking $N=2$. Moreover, given that current distribution along each metallic structure does not generate an electric field that could strongly interfere with the port of the neighbor antenna, the "no coupling losses" is a valid assumption. Hence, a perfectly matched and lossless pair of crossed-loop antennas can be said to be an efficient array.

If we restrict to the $x y$-plane, the corresponding RP vector simplifies to

$$
\mathbf{h}\left(\frac{\pi}{2}, \phi\right)=\left[\begin{array}{c}
\cos \phi \\
\sin \phi
\end{array}\right] .
$$

In this case, it is easier to find a transformed array whose response can distinguish impinging signals by phase instead of amplitude differences. Its array response can be translated into the RP vector that resembles the one corresponding to a set of two identical isotropic elements aligned to the $y$-axis and centered at the origin, that is

$$
\widetilde{\mathbf{h}}\left(\frac{\pi}{2}, \phi\right)=\left[\begin{array}{c}
e^{-j \phi} \\
e^{+j \phi}
\end{array}\right],
$$

and which is performed by means of the following transformation matrix 


$$
W=\frac{1}{\sqrt{ } 2}\left[\begin{array}{cc}
1 & j \\
1 & -j
\end{array}\right] .
$$

The RP vector in Equation 15 changes the way that phase argument varies according to $\varphi$ in comparison to a phased array whose RP vector is

$$
\mathbf{h}^{\circ}\left(\frac{\pi}{2}, \phi\right)=\left[\begin{array}{c}
e^{-j \frac{\pi}{2} \sin \phi} \\
e^{+j \frac{\pi}{2} \sin \phi}
\end{array}\right]
$$

However, the phase argument $-\frac{\pi}{2} \leq \phi \leq \frac{\pi}{2}$ varies in the same range as an array of two isotropic elements separated by $d=\frac{\lambda}{2}$ do, that is, $-\frac{\pi}{2} \leq \frac{\pi}{2} \sin \phi \leq \frac{\pi}{2}$. This fact puts in evidence that their behaviors are not far from each other, neither to the crossed-loop antennas. Hence, a strategy for finding equivalent arrays by means of an orthonormal transformation is needed and will be proposed in the next section.

\section{ORTHONORMAL TRANSFORMATION METHOD}

The objective of the proposed method is to compensate the actual AA response $\mathbf{x}$, transforming it into the signal vector $\tilde{\mathbf{x}}$, whose new structure can be described by some equivalent array response. From Equation 3 we can express

$$
\widetilde{\mathbf{x}}[m]=W H \mathbf{y}[m]+W \mathbf{e}[m]=\widetilde{H} \mathbf{y}[m]+\widetilde{\mathbf{e}}[m],
$$

where $W$ is the sought for unitary matrix transformation and $\tilde{H}$ is the new equivalent array matrix response, as close as possible to the $H^{\circ}$ desired response.

The actual array response matrix $H$ can be constructed based on DOAs of $P$ impinging signals at every instant $m$ for real-time processing, or can be pre-defined for a generic set of $P$ DOAs of interest as a static stage. In the first case, the advantage of the transformation can be an increase in the algorithmic precision if DOAs and array responses are well known, but it can require high signal processing capabilities. The second case reduces computational complexity by resigning precision because of performing a global transformation.

Figure 3 shows a block diagram of a generic GNSS receiver with a transformation stage for compensation of the individual AA responses at a given DOA $(\theta, \varphi)$.

Regarding the noise terms, when thermal agitation is the main source, the vector e of $N$ random variables can be assumed as complex normally distributed, being mutually independent and also to signals of interest, and identically distributed along the received signal vector, that is, $\widetilde{\mathbf{x}}=\mathcal{C N}\left(H \mathbf{y}, \sigma^{2} I_{N}\right)$, with $\sigma^{2}$ the noise variance. After applying the transformation matrix $W$, it is desirable that the resulting noise vector $\widetilde{\mathbf{e}}$ inherits not only the same probability distribution but also preserves independence so as not to affect

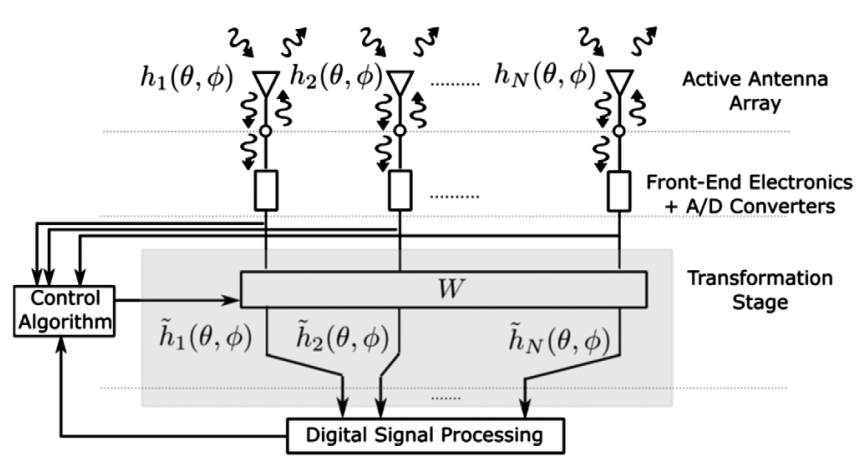

FIGURE 3. Global Navigation Satellite Systems receiver model

signal processing in the next stages. By constraining $W$ to be an unitary matrix $\left(W^{H} W=W W^{H}=I_{N}\right)$, the original input covariance structure is not modified, that is,

$$
\begin{array}{r}
\operatorname{Cov}\{\mathbf{x}\}=E\left\{\mathbf{x} \mathbf{x}^{H}\right\}-E\{\mathbf{x}\} E\left\{\mathbf{x}^{H}\right\}=\sigma^{2} I_{N}, \\
\operatorname{Cov}\{\tilde{\mathbf{x}}\}=W \operatorname{Cov}\{\mathbf{x}\} W^{H}=W \sigma^{2} I_{N} W^{H}=\sigma^{2} I_{N},
\end{array}
$$

where the superscript $H$ represents Hermitian transpose, $E$ $\{\cdot\}$ and $\operatorname{Cov}\{\cdot\}$ are the expectation and covariance operators respectively.

Moreover, if an unitary matrix $W$ is applied to $\mathbf{x}$, the total received power remains unaltered, that is,

$$
\widetilde{P}=\widetilde{\mathbf{x}}^{H} \widetilde{\mathbf{x}}=\mathbf{x}^{H} W^{H} W \mathbf{x}=P_{\text {total }},
$$

and Equation 6 now holds for the new excitation vector $\tilde{\mathbf{x}}$ resulting in

$$
\widetilde{\mathbf{x}}^{H}\left(I_{N}-S^{H} S\right) \widetilde{\mathbf{x}} \geq \widetilde{\mathbf{x}}^{H} \Gamma \widetilde{\mathbf{x}}
$$

In the following, a method for solving this constrained optimization problem based on the OPP will be presented.

\subsection{Orthogonal Procrustes problem}

A matrix $W$ that belongs to a $N \times N$ unitary group over a complex field, $W \in U(N, C)$, performs rotations on some $\mathrm{N}$-dimensional element inside the subspace it is contained. The traditional problem of finding the closest matrix to another through a linear transformation that only admits rotations over the $N$-dimensional subspace-in Euclidean distance-is called the OPP. ${ }^{15}$ The transformation $W$ can be found by minimizing the following cost function

$$
\gamma=\left\|\left(W H-H^{\circ}\right) Q\right\|_{F}^{2} .
$$

where $\|\cdot\|_{F}$ is the Frobenius norm.

The problem posed in Equation 23 is the classical weighted OPP (WOPP) and has a closed-form solution. This formulation includes a real positive definite diagonal weight matrix $Q$ which allows making different penalizations according to any chosen DOA. For a global compensation it 
can be set as an identity matrix, but it can be useful when performing compensation on a reduced number of DOAs.

Calling $B=H Q$ and $A=H Q^{\circ}$, with $A, B \in \mathrm{C}^{N \times P}$, and expanding Frobenius norm by means of matrix trace, it is found that

$$
\begin{gathered}
\gamma=\|W B-A\|_{F}^{2}= \\
=\operatorname{tr}\left\{B^{H} W^{H} W B\right\}+\operatorname{tr}\left\{A^{H} A\right\}-2 \operatorname{tr}\left\{B^{H} W^{H} A\right\} .
\end{gathered}
$$

Noting that only the third term depends on $W$, the optimal solution with respect to this variable is obtained by maximizing the opposite of this term. Introducing the singular value decomposition (SVD) of $A B^{H}=U \Sigma V^{H}$ yields

$$
\begin{gathered}
\operatorname{tr}\left\{B^{H} W^{H} A\right\}=\operatorname{tr}\left\{A B^{H} W^{H}\right\}= \\
=\operatorname{tr}\left\{U \Sigma V^{H} W^{H}\right\}=\operatorname{tr}\left\{\Sigma V^{H} W^{H} U\right\},
\end{gathered}
$$

where $\Sigma=\operatorname{diag}\left(\left[\sigma_{1}, \sigma_{2}, \ldots, \sigma_{\mathrm{P}}\right]\right)$, with singular values $\sigma_{\mathrm{p}} \geq 0$, is a real positive semi-definite diagonal matrix and $V^{H} W^{H} U$ is a new unitary matrix. Hence, it can be proved that Equation 25 maximizes when the unitary matrix $W$ equals to. ${ }^{15}$

$$
W_{\text {opt }}=\underset{W}{\operatorname{argmax}}\left\{\operatorname{tr}\left\{\Sigma V^{H} W^{H} U\right\}\right\}=U V^{H},
$$

which yields

$$
\because \max _{\mathrm{W}}\left\{\operatorname{tr}\left\{\Sigma \mathrm{V}^{\mathrm{H}} \mathrm{W}^{\mathrm{H}} \mathrm{U}\right\}\right\}=\operatorname{tr}\{\Sigma\} .
$$

Therefore, Equation 26 is the closed-form solution for $W$ that can be found in terms of the SVD of the $A B^{H}=$ $H^{\circ} Q Q^{H} H^{H}$ matrix.

\subsection{Correlation function of AAs}

Previous results show how an optimal solution to the WOPP can be found for a given array response at DOAs of interest. Now, we are interested in finding a transformation as exact as possible. Hence, for a given objective AA response $H^{\circ}$, it is necessary to propose a properly designed array response $H$ so that the degrees of freedom of an orthonormal matrix $W$ are enough to reduce the magnitude of Equation 23 as much as possible.

Employing transformation Equation 26 and according to the desired DOAs that determine $H$ and $H^{\circ}$, the matrices $A$ and $B$ can be expressed in terms of the SVD, which delivers an orthonormal base of left and right eigenvectors of both matrices besides its own singular values

$$
A=U_{A} \Sigma_{A} V_{A}^{H} ; B=U_{B} \Sigma_{B} V_{B}^{H} .
$$

Eigenvector bases $U_{A}$ and $U_{B}$ belong to the same vector subspace, in the same way as $V_{A}$ and $V_{B}$ do. Our tool is an unitary matrix that performs a linear transformation (rotation) over the left eigenvector base and it can be applied to get $W U_{B}=U_{A}$ whereas the rest is not modified. Hence, a good fit will be obtained if eigenvalues and right eigenvectors are the same, that is, $\Sigma_{A}=\Sigma_{B}$ and $V_{A}=V_{B}$.
Or equivalently, it should be verified that matrices $R_{A}=$ $A^{H} A$ and $R_{B}=B^{H} B$ match perfectly, that is,

$$
R_{B}=B^{H} B=A^{H} A=R_{A} .
$$

If this condition is verified, then Equation 28 becomes.

$$
A=U_{A} \Sigma_{A} V_{A}^{H} ; B=U_{B} \Sigma_{A} V_{A}^{H},
$$

which allows the SVD of $A B^{H}=U_{A} \Sigma_{A}^{2} U_{B}^{H}$ to be achievable. In this condition, Equation 26 results $W_{\text {opt }}=U_{A} U_{B}^{H}$, and consequently Equation 23 equals to

$$
\gamma_{0}=\left\|\left(U_{A} U_{B}^{H}\right)\left(U_{B} \Sigma_{\mathrm{A}} V_{A}^{H}\right)-U_{A} \Sigma_{A} V_{A}^{H}\right\|_{F}^{2}=0 .
$$

Matrices $B$ and $A$ are respectively defined by the actual array and the objective array characteristics correspondingly. Therefore, it is insightful to make a proper design of both so that Equation 29 is met as close as possible. Actually, Equation 29 establishes a necessary and sufficient condition to satisfy $\gamma=0$, so that an exact transformation can be obtained for every design of $H$ and $H^{\circ}$ that meets Equation 29. That allows us to settle the array design problem in terms of the minimization of the new cost function $\gamma^{p}$,

$$
\gamma^{p}=\left\|A^{H} A-B^{H} B\right\|_{F}^{2}=\left\|Q^{H}\left[H^{H} H-H^{\circ} H^{\circ}\right] Q\right\|_{F}^{2} .
$$

Expanding Equation 32 in terms of the RP vectors, the sought for array design constraint becomes clearer

$$
\left\langle\mathbf{h}_{i}, \mathbf{h}_{k}\right\rangle \approx\left\langle\mathbf{h}_{i}^{\circ}, \mathbf{h}_{i}^{\circ}\right\rangle,
$$

where $\left\langle\mathbf{h}_{i}, \mathbf{h}_{k}\right\rangle=\mathbf{h}_{i}^{H} \mathbf{h}_{k}$ is the inner product $\forall i, k=1, \ldots, P$.

From Equation 33, it can be concluded that a necessary condition for this transformation method to be exact is that, for every pair of desired DOAs, the vector correlation remains equal between actual and objective arrays. If we generalize this for all possible pairs of DOAs $\left(\theta^{\prime}, \varphi^{\prime}\right)$ and $\left(\theta^{\prime \prime}, \varphi^{\prime \prime}\right)$, a new four-dimensional correlation function $r$ can be defined

$$
r\left(\theta^{\prime}, \varphi^{\prime}, \theta^{\prime \prime}, \varphi^{\prime \prime}\right)=\left\langle\mathbf{h}\left(\theta^{\prime}, \varphi^{\prime}\right), \mathbf{h}\left(\theta^{\prime \prime}, \varphi^{\prime \prime}\right)\right\rangle .
$$

This function has lot of valuable information about the characteristics of AAs. It maps correlation of RP vectors $h$ for all possible combinations of DOA variables. Its magnitude indicates how collinear two particular vectors are at two different DOAs. If these two orientations are highly correlated, they can be easily confused when, for example, performing DOA estimation. The optimal case would be perfect orthogonality, while maximum correlation points out that both directions are indistinguishable by their respective RP vectors. Moreover, in the case of performing interference rejection, the information of how much it will affect to put a null on interference DOA to the maximum achievable gain over signal of interest DOA is also contained in the correlation function $r$. Many AA applications can be interpreted by means of the correlation function $r$ because it has information about all possible DOAs dependencies. It describes the capacity of an AA to discriminate every pair of orientations 
in far-field. In consequence, two different array configurations will be declared equivalent, in terms of an unitary matrix transformation, if they have the same correlation function.

\subsection{Example 1 Revisited}

Recall the previously described example of Section 2.2. We analyze the RP only over the $x z$-plane so that the correlation function (34) can be plotted. It will be analyzed over the two elevation variables $\theta^{\prime}$ and $\theta^{\prime \prime}$, obtaining the following expression

$$
r\left(\theta^{\prime}, \theta^{\prime \prime}\right)=\sum_{n=-\frac{N}{2}}^{\frac{N}{2}-1} e^{j \frac{d \pi}{\lambda}(2 n+1)\left[\sin \left(\theta^{\prime \prime}\right)-\sin \left(\theta^{\prime}\right)\right]}
$$

In Figure 4 the absolute value of the correlation function $\left|r\left(\theta^{\prime}, \theta^{\prime \prime}\right)\right|$ is compared for two isotropic antennas according to their separation $d$. For every point in the graph we can appreciate how correlated is the vector $\mathbf{h}\left(\theta^{\prime}\right)$ to $\mathbf{h}\left(\theta^{\prime \prime}\right)$. It can be seen that for two antennas at short distances the resolution is very poor, but it improves with increasing distance. However, if distance increases too much, ambiguity appears, like in the case of $d=\lambda$ where maximum correlation occurs at least twice when moving horizontally or vertically on an imaginary line in graph. For a pair of ideally identical antennas separated $d=\frac{\lambda}{2}$ there exist maximum resolution without ambiguity (except at end-fire condition, ie, null elevation), which is usually taken as the traditional rule of design.

In Figure 5 the absolute value of the correlation function $\left|r\left(\theta^{\prime}, \theta^{\prime \prime}\right)\right|$ is compared for $N$ isotropic antennas with pairwise separation $d=\frac{\lambda}{2}$. Now, with an increase in the number of antennas, the array aperture increases and it has the advantage of expanding the low amplitude region in correlation function map. Besides, the resolution increases too, which is considered as the distance from some point at the principal diagonal $\left(\varphi^{\prime}=\varphi^{\prime \prime}\right)$ to the first correlation null when moving horizontally or vertically. In the cases analyzed earlier, their different capabilities can be clearly described by means of their correlation functions. They all have proper correlation functions and they represent important characteristics of these AAs of ideal isotropic elements in this case.

(A)

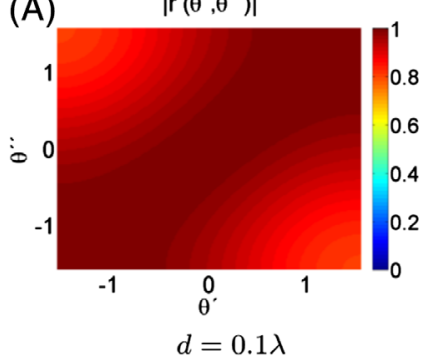

(B)

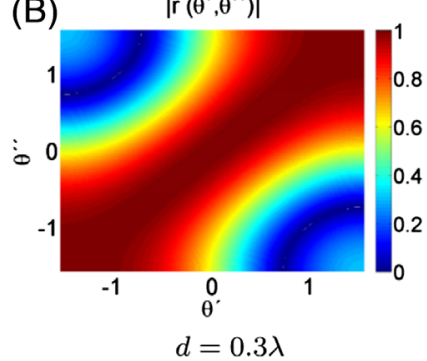

\subsection{Example 2 Revisited}

We now reconsider the example proposed in Section 2.3 of an AA of the crossed-loop antennas and a similar $\frac{\lambda}{2}$-spaced phased array. Figure 6 compares correlation function $r\left(\varphi^{\prime}, \varphi^{\prime \prime}\right)$ for both cases mentioned above. It can be seen that both functions are quite similar but there are some changes about their resolution. Crossed-loop antennas have equal resolution at every reference point and it is $\frac{\pi}{2}$. On the contrary, in the case of the two element phased array, resolution changes according to reference DOA, being $\frac{\pi}{2}$ at $\phi^{\prime}=-\frac{\pi}{2}, 0, \frac{\pi}{2}$; and taking smaller values in between.

Some other array designs may have neither the same nor similar correlation functions that correspond to a realizable AA of identical antennas. Then, the equivalent arrays that can be obtained by means of the unitary transformation could be understood as virtual phased arrays with different characteristics and, perhaps, with some improved performance at certain DOAs. Therefore, this method can be highly promising for efficient compact array designs.

\section{EFFICIENT COMPACT MICROSTRIP AA}

Typically, GNSS receivers employ microstrip antennas since they are characterized by having good hemispherical radiation properties, which is important to maximize the visibility range of available satellites. In addition, microstrip antennas can be easily designed to achieve circular polarization, they are low cost, mechanically robust and have low profile. In particular, in this work a microstrip AA of square patches with truncated corners was selected to obtain right hand circular polarization (RHCP). ${ }^{9}$

\subsection{Array design}

Each antenna has a single coaxial feed and is implemented on a (Rogers Corporation) RT/duroid 6002 substrate with relative dielectric permittivity $\varepsilon_{r}=2.94 .^{22}$ For the array structure, a disposition of two linearly aligned antennas separated by a distance of $d=0.3 \lambda$ with same substrate and ground plane is proposed, being $\lambda$ the wavelength of the center frequency of the L1 band of the Global Positioning System (GPS), $f_{\mathrm{c}}=1.57542 \mathrm{GHz}$. The AA and its parameters
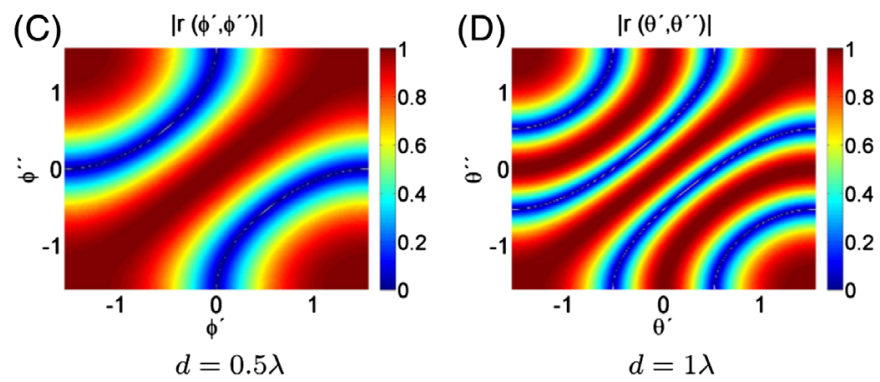

FIGURE 4. Correlation function of a phased array of two antennas 

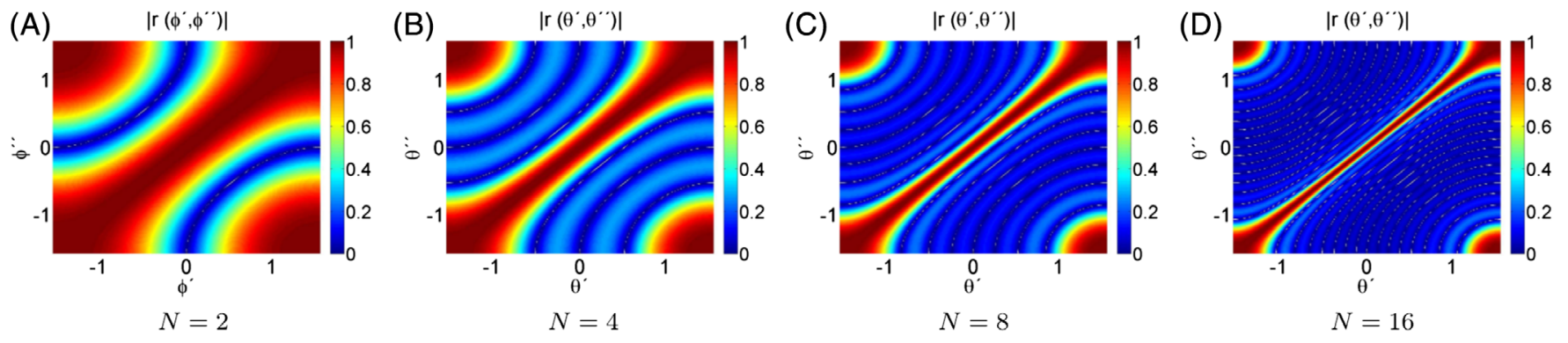

FIGURE 5. Correlation function of a phased array with inter-element distances $d=\frac{\lambda}{2}$
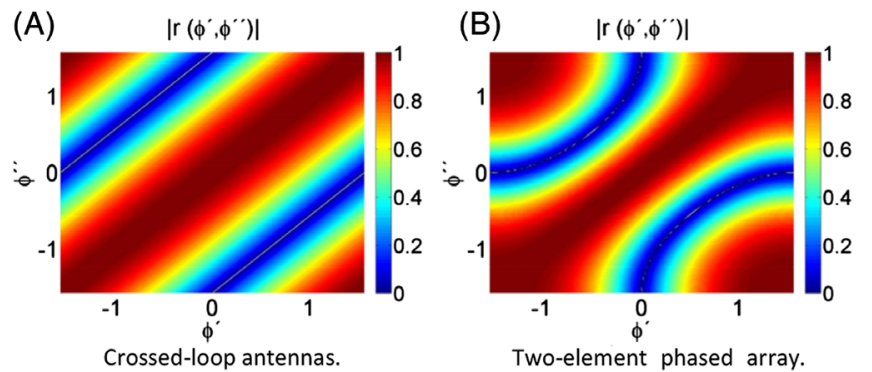

TABLE 1. Antenna dimensions

\begin{tabular}{ll} 
Parameter & Dimension $(\mathbf{m m})$ \\
\hline$c$ & 5.4 \\
$h$ & 13.6 \\
$g$ & 150 \\
$l$ & 100 \\
\hline$e$ & 54.6 \\
\hline$t$ & 1.524 \\
\hline
\end{tabular}

FIGURE 6. Comparison of the correlation function of the crossed-loop antennas and a two element $\frac{\lambda}{2}$-spaced phased array

of interest are shown in Figure 7. The selected parameters, as well as distance and disposition between array elements were chosen to reduce the impedance mismatch and coupling losses.

Final dimensions of the already described microstrip AA were obtained by means of computer-aided simulations and are presented in Table 1.

\subsection{Array response}

The simulated AA was tuned to be matched at $f_{\mathrm{c}}$ with a bandwidth of $16 \mathrm{MHz}$ where the scattering parameters are all below the threshold given by $\left|S_{i k}\right|<-10 \mathrm{~dB}$ as it can be seen in Figure 8A. Matching and coupling losses are equal between antennas because of the rotational symmetry of the array structure. It is also important to note the frequency

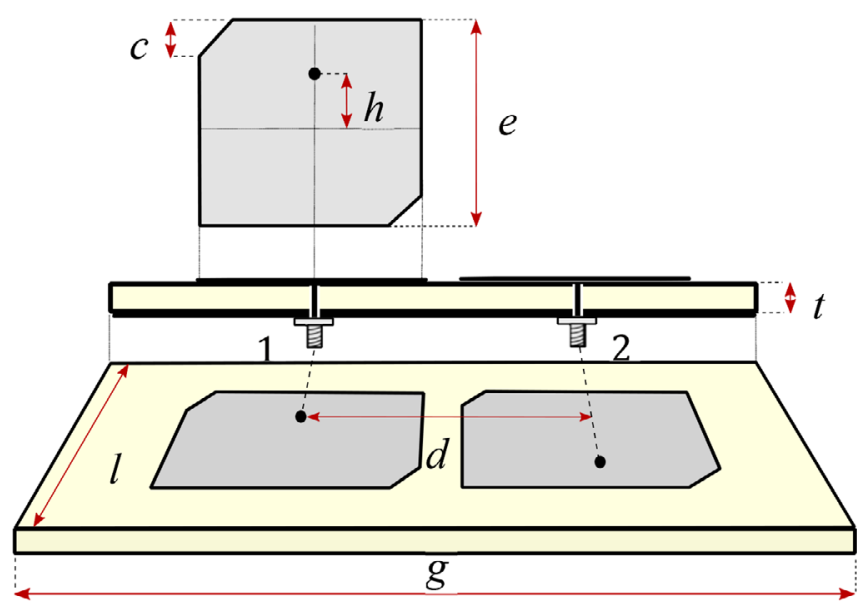

FIGURE 7. Patch antenna element and two-element linear array

variation of $\left|S_{21}\right|$ because it shows that a considerable amount of received power is reflected except at $f_{\mathrm{c}}$ surroundings. Despite the active antenna mutually exciting the passive one, their current distributions are not the same. While the active antenna tends to have an adequate current distribution for RHCP, the behavior of the second antenna is described according to MCED effects. Particularly at $f_{\mathrm{c}}$ frequency, the current distribution in the passive antenna is mostly horizontally aligned. Consequently, the resultant electric field seen at the passive port is reduced in magnitude and, correspondingly, the mutual coupling parameter $\left|S_{21}\right|$ is nearly null.

In Figure 8B, the radiation efficiency of each element $\left(\eta_{i}, i=1,2\right)$ is shown in pink. Conversely, the purple curve represents total efficiency $\left(\epsilon_{i} \eta_{i}, i=1,2\right)$, taken as the product of radiation and reflection efficiencies, so the pink curve represents an upper bound to the total achievable efficiency. It can be seen that the reflection losses are almost negligible at $f_{\mathrm{c}}$ and its surroundings because both curves are nearly equal in the range where all $\left|S_{i k}\right|$ parameters are under the $-10 \mathrm{~dB}$ level. Moreover, the maximum total efficiency is achieved at $f_{\mathrm{c}}$ with a value of $-0.5 \mathrm{~dB}$, and also keeps above $-1 \mathrm{~dB}$ within a range of $20 \mathrm{MHz}$, being the array more than adequate for the GPS
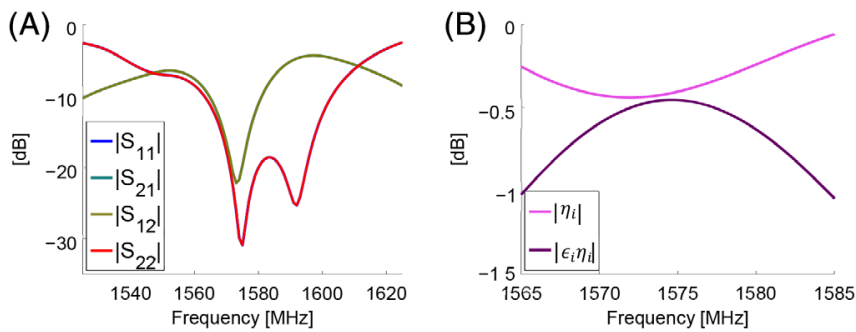

FIGURE 8. Array efficiency simulated results 

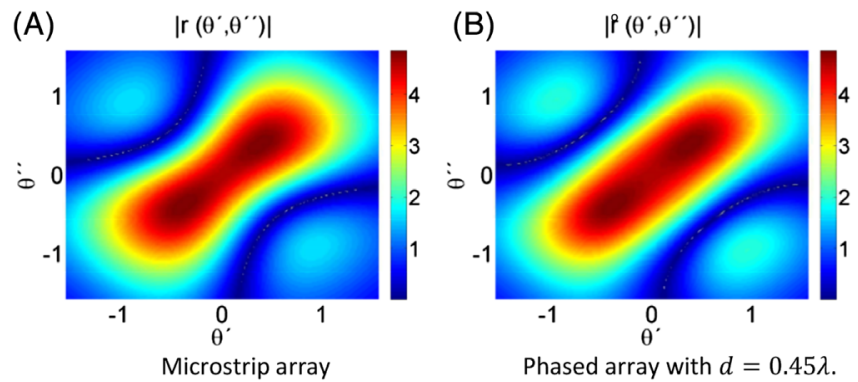

FIGURE 9. Correlation function comparison of the designed microstrip antenna array and the objective array

signal bandwidth requirements. It is important to remark that the total efficiency achieved by this AA example is higher than many recently proposed compact designs. $4,5,17,18,23,24$ The main reason is that these array designs are intended to reduce reflected power at the expense of creating a lossy structure by, for example, employing high dielectric permittivity, fences, resonant structures, or decoupling and matching networks, which actually creates an inefficient array system. However, as we have previously described, it is not necessary to make use of these elements if our approach is adopted.

The correlation function in the $x z$-plane $-\left|r\left(\theta^{\prime}, 0^{\circ}, \theta^{\prime \prime}, 0^{\circ}\right)\right|-$ of the microstrip array is shown in Figure 9A. It shows that at the zenith region the DOAs are received with greater amplitude than at end-fire region. As it was previously discussed, the sought for objective array must be selected according to the correlation function of the designed array and selected DOAs. In this case, a grid was defined in the range $0^{\circ} \leq \theta \leq 90^{\circ}$ and $0^{\circ} \leq \varphi<360^{\circ}$ with a step angle of $5^{\circ}$, resulting in 1368 different DOAs for the fitting purposes. Considering a desired virtual array that behaves like equal and uncoupled antennas, Equation 33 suggests that an adequate common RP for the objective array needs to satisfy

$$
\left\langle\mathbf{g}_{i}, \mathbf{g}_{\mathrm{i}}\right\rangle \approx\left\langle\mathbf{g}_{i}^{\circ}, \mathbf{g}_{i}^{\circ}\right\rangle
$$

which means that the RP gain at each of the selected DOAs should be equal to the root mean square of the actual AA gain patterns. Moreover, given its apparent resolution capacity indicated by the shape of the null correlation function region-a similar behavior is achieved with a pair of identical antennas separated by a distance $d=0.45 \lambda$. Their correlation function is presented in Figure 9B. Similarities of the two-element $0.3 \lambda$ separated microstrip array to an ideal phased array of two identical elements separated by $0.45 \lambda$ are evident, and then, a good transformation is possible.

In Figure 10, the upper hemisphere of RHCP RPs of the microstrip antennas are presented. Side lobes at lower hemisphere are not relevant since ground plane dimensions are big enough to moderate their magnitude. It can be noticed that due to the high coupling between antennas, their individual RPs have increased their directivity to different orientations, and such differences offer the proper conditions for almost orthogonal RPs. In Figure 11, the RHCP RPs of the proposed objective array are shown. Equal gain amplitude and the characteristic phase response of a phased array of two $0.45 \lambda$ separated elements is presented. Finally, the resultant RPs after the unitary transformation are shown in Figure 12. The RPs of the equivalent array can achieve very similar responses compared to the proposed phased array as predicted with the correlation function in Figure 9.
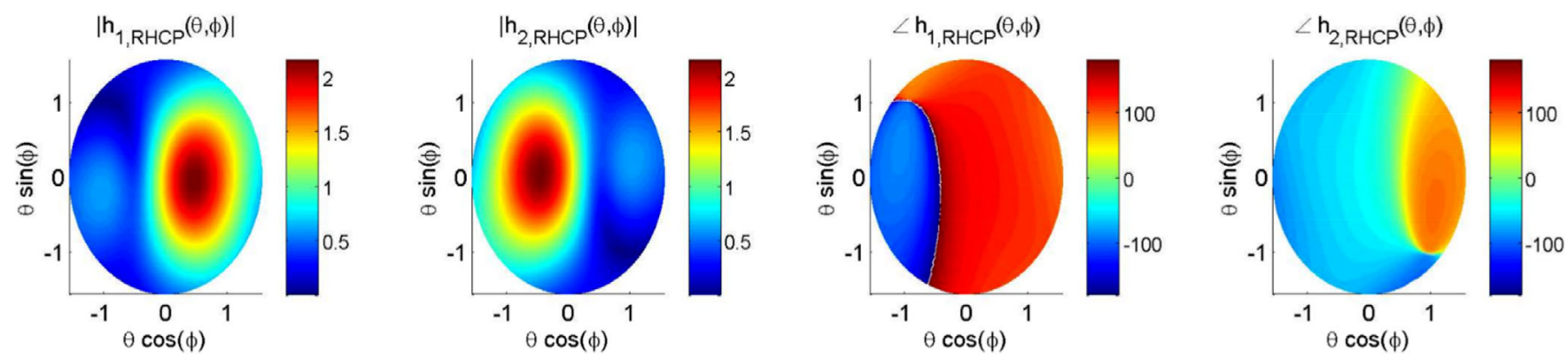

FIGURE 10. Resulting right hand circular polarization radiation patterns of the designed $0.3 \lambda$ separated microstrip array
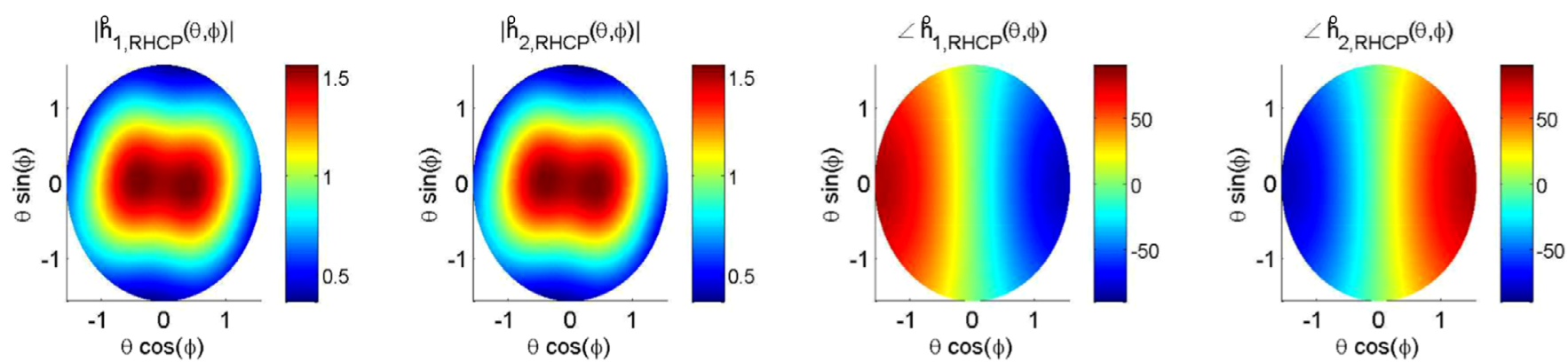

FIGURE 11. Right hand circular polarization radiation pattern responses of the $0.45 \lambda$ separated objective array 

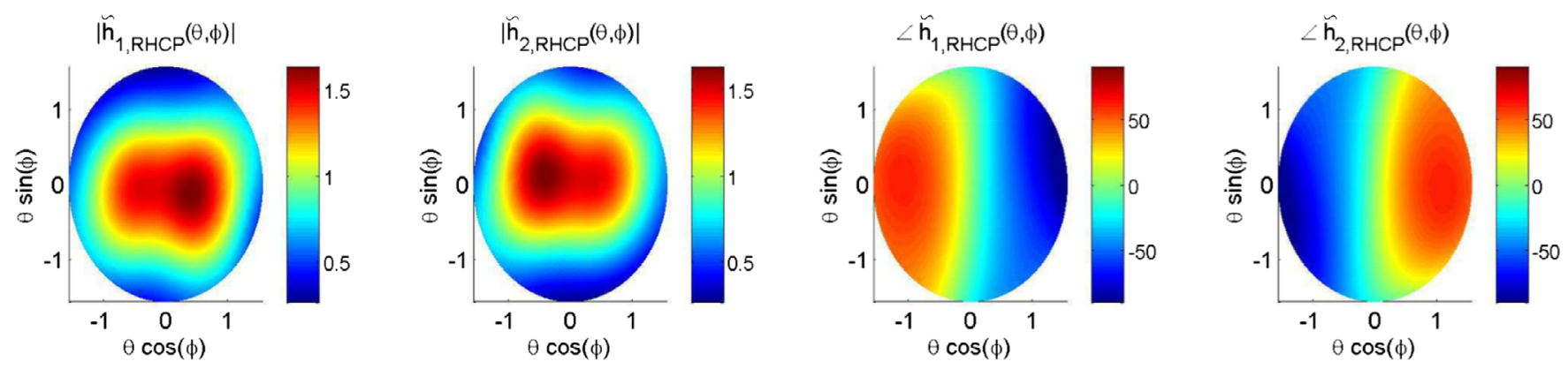

FIGURE 12. Transformed array right hand circular polarization radiation pattern responses
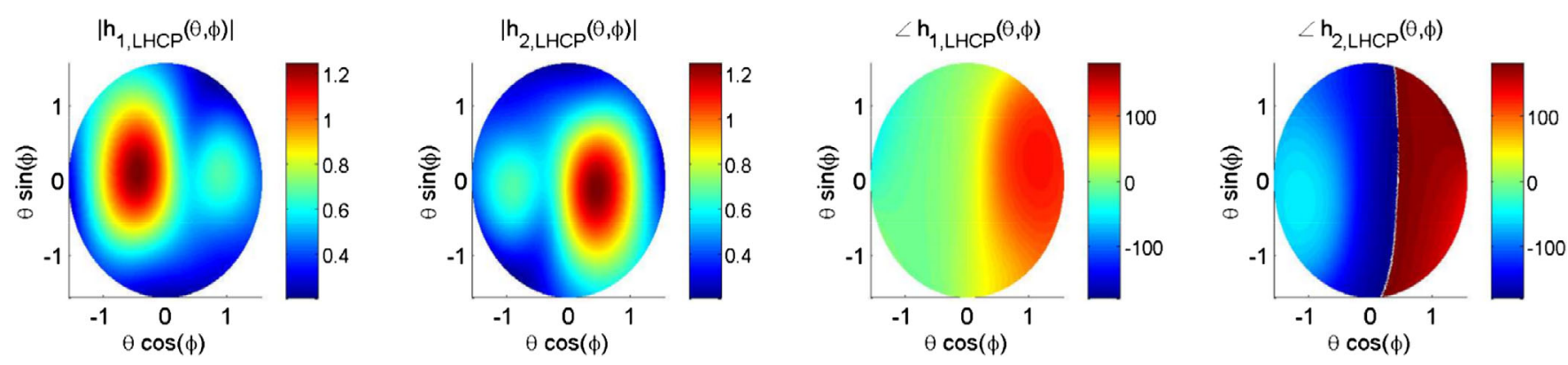

FIGURE 13. Resulting left hand circularly polarized radiation patterns of the designed array

Joint current distribution of the pair of coupled antennas provides previously described RHCP RPs. As a consequence, the left hand circularly polarized (LHCP) RP changes its behavior in comparison to an isolated antenna too. Figure 13 presents the LHCP RP of the designed AA. Its polarization efficiency is high for some DOAs but low for some others. It is a simple array that has the advantage of ease of implementation but a more detailed and complex geometry could improve the polarization efficiency by redirecting the overall current distribution. Considering that we used single feed patch antennas, an increase in polarization purity could be achieved by means of a dual port feeding network. In Figure 14 the resultant LHCP RPs of the transformed array are shown. Changes about their directivity can be noticed as well as different behaviors about their phase patterns. However, given that the transformation matrix fulfills the condition of being unitary, it does not make changes in the amount of total power that an array is able to receive at each DOA, even though LHCP RPs can change in shape.
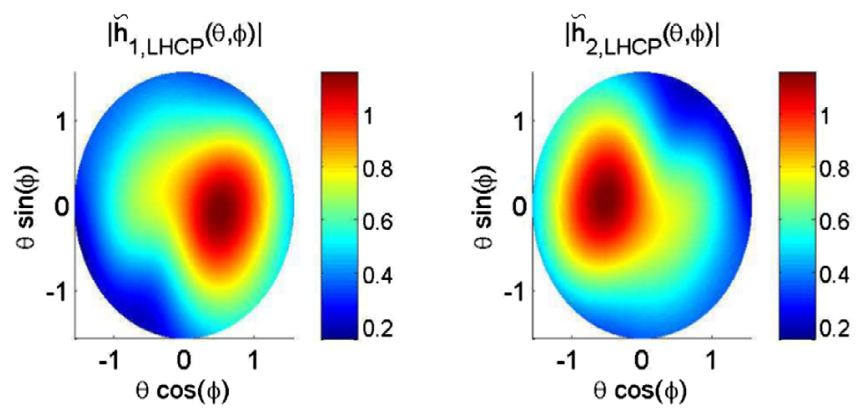

The compact GNSS AA design presented is an efficient radiating structure with simple reconfiguration capabilities given that its behavior can be translated to an equivalent ideal phased array to ease further signal processing stages. More sophisticated smart antennas can be designed based on this proposed technique for compact arrays. In particular, in future developments polarization purity will be addressed. However, even in the case that the proposed compact array is just an example of the proposed design method, it is already much more efficient than traditional arrays that tries to force the common RP rule by design as inter-element distance decreases. ${ }^{3-5}$

\section{CONCLUSIONS}

In this work we have presented a new compact AA design method based on orthogonal in situ RPs rather than equal patterns, and a unitary transformation that allows to use it as if it were a $\frac{\lambda}{2}$-spaced array with equally behaved patterns.
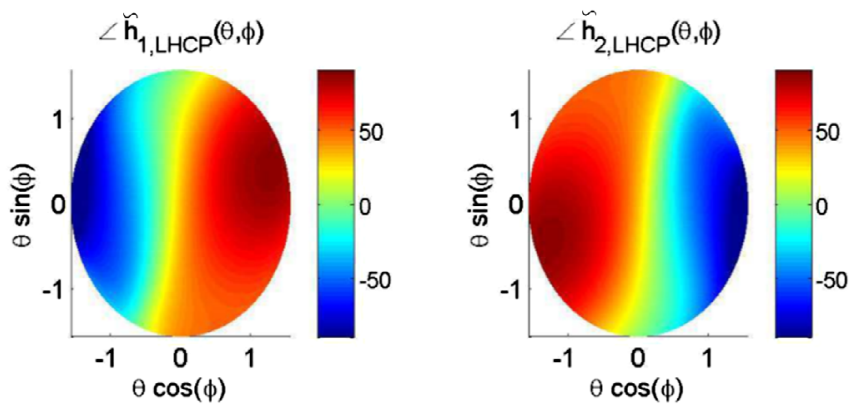

FIGURE 14. Transformed array left hand circularly polarized radiation pattern responses 
Orthogonal in situ RPs is a necessary condition to avoid losses and therefore to have an efficient compact AA. To ensure this orthogonality, joint phase and magnitude differences in RPs should be considered for array design. A proper design can accomplish these objectives, as shown with a computer-aided AA example, so that it has enough spatial diversity improvement in comparison to an ideally behaved compact phased array design. Hence, complex information about RPs should be determined by simulation/calibration, stored, and properly employed at signal processing stages. Therefore, the proposed orthonormal array design and signal processing method that allows to translate an efficient compact AA into an equivalent phased array which must be described by sharing the same correlation function. An array design should have an adequate correlation function to behave efficiently. In addition, some of its properties could be enhanced or relaxed within several design considerations as, for example, array resolution optimization according to DOAs of interest. The correlation function of an actual array design is not necessarily restricted to be fitted by a phased array. The objective array may be completely defined by user specifications so far as it fits such function.

The proposed method is meant to reduce array signal processing complexity of smart antennas by being a nexus between actual compact AAs designs and classical phased arrays. Several designs for efficient compact arrays, without restriction in the number of elements, can be considered for smart AAs by employing the proposed technique. In spite of the application example is being defined for GNSS, the proposed method is clearly not restricted for GNSS array designs only. The general assumption is that signals fit to a narrowband model, so that array RPs can be considered constant along the bandwidth. A generalization of the proposed method to other applications where a narrowband model does not apply is also possible and it will be addressed by the authors in the future.

\section{ACKNOWLEDGMENTS}

This work was supported by the following institutions: Universidad Nacional de La Plata (UNLP) and Agencia Nacional de Promoción Científica y Tecnológica (ANPCyT).

\section{REFERENCES}

1. Kaplan E, Hegarty CJ. Understanding GPS. Principles and Applications. 2nd ed. Boston, London: Artech House; 2006.

2. Gupta IJ, Weiss IM, Morrison AW. Desired features of adaptive antenna arrays for GNSS receivers. Proc IEEE. 2016;104(6):1195-1206.

3. Volakis JL, O'Brien AJ, Chen CC. Small and adaptive antennas and arrays for GNSS applications. Proc IEEE. 2016;104(6):1221-1232.

4. Irteza S, Schäfer E, Sgammini M, Stephan R, Hein M. FourElement compact planar antenna Array for robust satellite navigation systems. EuCAP. 2013;19-23.

5. Caizzone S. Miniaturized E5a/E1 antenna Array for robust GNSS navigation. IEEE Antennas Wirel Propag Lett. 2017;16:485-488.

6. Marranghelli EA, La Valle GRL, Roncagliolo PA. Mutual coupling and electromagnetic diffraction influence on GPS microstrip antenna arrays. Paper presented at: 16th Workshop on Information Processing and Control (RPIC) Córdoba, Argentina 2015, 2016.
7. Stein S. On cross coupling in multiple-beam antennas. IRE Trans Antennas Propag. 1962;10:548-557.

8. Ludwig AC. Mutual coupling, gain, and directivity of an Array of two identical antennas. IEEE Trans Antennas Propag. 1976;24:837-841.

9. Balanis CA. Antenna Theory: Analysis and Design. Hoboken, NJ: Wiley; 2005.

10. Akçakaya M, Muravchik $\mathrm{CH}$, Nehorai A. Biologically inspired coupled antenna array for direction-of-arrival estimation. IEEE Trans Signal Process. 2011;59(10):4795-4808.

11. Henault $\mathrm{S}$, Antar Y. Unifying the theory of mutual coupling compensation in antenna arrays. IEEE Antennas Propag Mag. 2015;57(2):104-122.

12. Kim K, Sarkar TK, Palma MS. Adaptive processing using a single snapshot for a nonuniformly spaced array in the presence of mutual coupling and near-field scatterers. IEEE Trans Antennas Propag. 2002;50(5):582-590.

13. Daneshmand S, Sokhandan N, Zaeri-Amirani M, Lachapelle G. Precise calibration of a GNSS antenna Array for adaptive Beamforming applications. Sensors. 2014. [Online]. Available;9669-9691. www.mdpi.com/journal/sensors.

14. Mowlér M, Lindmark B, Larsson EG, Ottersten B. Joint estimation of mutual coupling, element factor, and phase center in antenna arrays. Hindawi, J Wirel Commun Netw. 2007;2007.

15. Horn R, Johnson C. Matrix Analysis. 2nd ed. New York: Cambridge; 2013.

16. Volmer C, Weber J, Stephan R, Blau K, Hein MA, Member S. An eigenanalysis of compact antenna arrays and its application to port decoupling. IEEE Trans Antennas Propag. 2008;56(2):360-370.

17. Qiao W, Gao X, Yu XY, Li SM, Jiang YN, Ma HF. Ultra compact microstrip antenna array and miniaturized feeding network. Prog Electromagn Res. 2017;71:111-122.

18. Yang $X M$, Liu $X G$, Zhou $X Y$, Cui TJ. Reduction of mutual coupling between closely packed patch antennas using Waveguided Metamaterials. IEEE Antennas Wirel Propag Lett. 2012;11:389-391.

19. Backén S, Akos D, Nordenvaad M. Post-processing dynamic GNSS antenna array calibration and deterministic beamforming. ION GNSS. 2008.

20. Daneshmand S, Jafarnia-jahromi A, Broumandan A, Lachapelle G. A lowcomplexity GPS anti-spoofing method using a multi-antenna array. ION GNSS. 2012.

21. Frater M, Ryan M. Electronic Warfare for the Digitized Battlefield. 1st ed. Boston, London: Artech House; 2001.

22. Rogers Corporation, RT/duroid 6002 High Frequency Laminates Data Sheet. Chandler, Arizona, USA. www.rogerscorp.com

23. Li J, Shi J, Feng K, Xiao Z, Chen J, Zhang A. Isolation enhanced circularly polarized patch antenna array using modified electric field coupled resonator. Int J RF Microw Comput Eng. 2018. https://onlinelibrary.wiley. com/doi/abs/10.1002/mmce.21560;e21560.

24. Gheethan AA, Herzig PA, Mumcu G. Compact $2 \times 2$ coupled double loop GPS antenna array loaded with broadside coupled split ring resonators. IEEE Trans Antennas Propag. 2013;61(6):3000-3008. 